\title{
Incidence and mortality of cervical cancer in China, 2013
}

\author{
Bingbing Song ${ }^{1,2^{*}}$, Chao Ding ${ }^{*}$, Wangyang Chen ${ }^{1,2}$, Huixin Sun ${ }^{1,2}$, Maoxiang Zhang ${ }^{1,2}$, Wanqing Chen ${ }^{3}$ \\ ${ }^{1}$ Tumor Prevention and Treatment Institute, Harbin Medical University, Harbin 150086, China; ${ }^{2}$ Heilongjiang Academy of Medical Sciences, \\ Harbin 150086, China; ${ }^{3}$ National Cancer Center/Cancer Hospital, Chinese Academy of Medical Sciences and Peking Union Medical College, \\ Beijing 100021, China \\ *These authors contributed equally to this work. \\ Correspondence to: Wanqing Chen, PhD. National Cancer Center/Cancer Hospital, Chinese Academy of Medical Sciences and Peking Union \\ Medical College, No. 17 Panjiayuan South Lane, Chaoyang District, Beijing 100021, China. Email: chenwq@cicams.ac.cn.
}

\begin{abstract}
Objective: Estimating the incidence and mortality rate of cervical cancer became necessary to establish prevention measures and healthy policies. The aim of this study was to estimate the updated incidence and mortality rate of cervical cancer in 2013 in China.

Methods: According to the evaluation criteria developed by the National Central Cancer Registry of China, the data submitted from 255 cancer registries met the required standards in 2013. Cervical cancer cases were retrieved from the national database and combined with the 2013 national population data. The age-standardized incidence and mortality rates were based on the demographic structure of the national census 2000 and Segi's world population.

Results: In 2013, the estimated number of new cases and deaths from cervical cancer were 100,700 and 26,400, respectively. The crude incidence of cervical cancer was 15.17/100,000. The age-standardized incidence rates based on the Chinese standard population (ASIRC) and the world standard population (ASIRW) were 11.30/100,000 and $10.30 / 100,000$, respectively. The incidence of cervical cancer in urban areas was 15.62/100,000 and the ASIRC was 11.12/100,000. The incidence of cervical cancer in rural areas was $14.65 / 100,000$ and the ASIRC was 11.47/100,000. The mortality rate of cervical cancer was 3.98/100,000. The age-standardized mortality rates based on the Chinese (ASMRC) and world standard populations (ASMRW) were 2.76/100,000 and 2.62/100,000, respectively. The mortality rate of cervical cancer in urban areas was 3.85/100,000 and in rural areas was 4.14/100,000. Cervical cancer incidence and mortality increased with age. Urban areas had a higher incidence of cervical cancer and lower mortality rates when compared with rural areas.

Conclusions: Dynamic monitoring of cervical cancer incidence and mortality is the fundamental work of cervical cancer prevention and control. Cervical cancer is a serious issue in women's health, and prevention strategies need to be enhanced, such as human papilloma virus (HPV) vaccination and screening programs.
\end{abstract}

Keywords: Cervical cancer; cancer registration; epidemiology

Submitted Jul 24, 2017. Accepted for publication Aug 25, 2017.

doi: 10.21147/j.issn.1000-9604.2017.06.01

View this article at: https://doi.org/10.21147/j.issn.1000-9604.2017.06.01

\section{Introduction}

Over the past 30 years, the increasing proportion of young women affected by cervical cancer has ranged from $10 \%$ to $40 \%$. According to the World Health Organization and International Agency for Research on Cancer estimates (1), the year 2008 saw 529,000 new cases of cervical cancer globally. In developing countries, the number of new cases of cervical cancer was 452,000 , and ranked second among malignancies in female patients. Conversely, the number of new cases of cervical cancer was 77,000 in developed countries, and ranked tenth among female malignancies. 
Per the Surveillance, Epidemiology, and End Results (SEER) data, the trend of both the incidence and mortality rate of cervical cancer showed a decline in the USA (2); however, the mortality rate showed an increasing trend in urban China (3). The highest incidence of cervical cancer was reported in Malawi and Zimbabwe, although the incidence is also high in parts of Central and South America. However, the incidence of cervical cancer was found to be lower in regions with a high human development index. The 5 -year survival rate among women diagnosed with cervical cancer varies greatly from $\geq 70 \%$ in developed countries to $<60 \%$ in low-income regions (4). Many factors such as social health inequities (5), place of residence, education (6), and human papilloma virus (HPV) vaccine coverage (7) have a strong impact on the incidence and survival rate of cervical cancer. Implementation of organized screening and prevention policies for cancer depends on high-quality cancer registry data. This study analyzed the incidence and mortality rate of cervical cancer based on the latest cancer registry data from whole country of China mainland in order to provide the updated epidemiological characteristic of cervical cancer for its prevention.

\section{Materials and methods}

\section{Cancer registration in China}

The National Central Cancer Registry (NCCR), National Cancer Center is in charge of population-based cancer registry in China (8), collecting cancer registration data from local registries. In 2016, 347 registries submitted data to NCCR were distributed in 31 provinces, autonomous regions, municipalities, and groups of the Xinjiang Production and Construction Corps including 126 cities (urban areas) and 221 counties and county-level cities (rural areas). Finally, 255 cancer registries were qualified for analysis, including 88 urban cities and 167 rural counties. The 255 cancer registries covered a population of $226,494,490(111,595,772$ in urban and $114,898,718$ in rural), including $114,860,339$ men and $111,634,151$ women, accounting for $16.65 \%$ of China population in the end of 2013.

\section{Evaluation of registration data}

NCCR evaluated the registration data based on the Guidelines for Chinese Cancer Registration (2016) (9), quality control criteria for the Cancer Incidence in Five Continents Volume IX (10) from the International Agency for Research on Cancer/International Association of Cancer Registry (IARC/IACR) (11). These data were reviewed and checked using MS-FoxPro, MS-Excel, SAS and IARCcrg Tools (12). The comparability, validity and timeliness of data were evaluated by indicators, including of the percentage of cases with morphological verification (MV) (\%), the percentage of death certificate only cases (DCO) (\%), mortality-to-incidence ratio (M/I) and the percentage of the diagnosis of unknown basis (UB) (\%).

The $M / I, M V \%$, DOC\% and UB\% were $0.27,86.59 \%$, $0.87 \%$ and 0.30 , respectively, which was concluded by evaluating the quality of pooled data from all accepted registries. The quality of data from urban and rural is shown in Table 1.

\section{Statistical analysis}

The incidence (mortality) rate, age-standardized incidence (mortality) rate, constituent ratio and cumulative rate of cervical cancer were calculated stratified by age, sex and area of residence. The standardized population used the sixth national census population in 2000 and Segi's world population.

\section{Results}

\section{Incidence of cervical cancer}

In 2013 , a total of 100,700 new cases of cervical cancer were estimated, accounting for $6.16 \%$ of malignancies in female patients. The crude incidence of cervical cancer was $15.17 / 100,000$. The age-standardized incidence rates by Chinese standard population (ASIRC) and by world standard population (ASIRW) were 11.30/100,000 and $10.30 / 100,000$, respectively, with a cumulative rate of $1.06 \%$ (0-74 years old). The crude incidence of cervical cancer, ASIRC and ASIRW were 15.62/100,000, $11.12 / 100,000$, and $10.08 / 100,000$ in urban areas, with a cumulative rate of $1.02 \%(0-74$ years old), and $14.65 / 100,000,11.47 / 100,000$ and $10.54 / 100,000$ in rural

Table 1 Quality control indices of cervical cancer in China, 2013

\begin{tabular}{lcccc}
\hline Area & $\mathrm{M} / \mathrm{I}$ & $\mathrm{MV}(\%)$ & $\mathrm{DOC}(\%)$ & $\mathrm{UB}(\%)$ \\
\hline Overall & 0.27 & 86.59 & 0.87 & 0.30 \\
Urban areas & 0.26 & 87.23 & 0.91 & 0.46 \\
Rural areas & 0.28 & 85.96 & 0.84 & 0.13 \\
\hline
\end{tabular}

$\mathrm{M} / \mathrm{I}$, mortality-to-incidence ratio; $\mathrm{MV}$, cases identified via morphological verification; $\mathrm{DOC}$, percentage of death certificateonly cases; UB, percentage of the diagnosis of unknown basis. 
areas, with a cumulative rate of $1.11 \%$ ( $0-74$ years old) (Table 2).

\section{Age-specific incidence of cervical cancer}

The age-specific incidence of cervical cancer increased with age. The increase in incidence reached a peak $(33.33 / 100,000)$ at age of 50 , but decreased annually $(11.86 / 100,000)$ at age $\geq 85$ years. The incidence rates of cervical cancer were higher in urban areas than in rural areas among patients aged $<50$ years. The incidence rates of cervical cancer in urban areas were lower than that in rural areas among those aged $\geq 50$ years. The incidence of cervical cancer in urban and rural areas reached a peak at age $\geq 45$ years $(34.24 / 100,000)$ and $\geq 50$ years (32.05/100,000), respectively (Figure 1).

\section{Mortality rate of cervical cancer}

It was estimated that the number of deaths due to cervical cancer was 26,400 in 2013 and accounted for $3.21 \%$ of cancer-related deaths among female patients. The mortality rate of cervical cancer was $3.98 / 100,000$. The age-standardized mortality rates by Chinese standard population (ASMRC) and by world standard population (ASMRW) were 2.76/100,000 and 2.62/100,000, respectively, with a cumulative rate of $0.29 \%$ ( $0-74$ years old) in 2013. The crude mortality rate of cervical cancer, ASMRC and ASMRW were 3.85/100,000, 2.56/100,000, and $2.41 / 100,000$ in urban areas, with a cumulative rate of $0.26 \%$ (0-74 years old), and 4.14/100,000, 2.99/100,000 and 2.87/100,000 in rural areas, with a cumulative rate of $0.33 \%$ (0-74 years old) (Table 3$)$.

\section{Age-specific mortality rate of cervical cancer}

The age-specific mortality rate of cervical cancer was low over the ages of 0-29 years. After 30 years old, the mortality rate of cervical cancer increased gradually with age, and reached a peak at age $\geq 85$ years $(15.96 / 100,000)$. The age-specific mortality rates of cervical cancer in both urban and rural areas were not different at age $\geq 50$ years. The age-specific mortality rate of cervical cancer in urban areas was higher than that in rural areas over the ages of $\geq 55$ to $\geq 75$ years. On the contrary, after age $\geq 80$ years, this trend reversed (Figure 2).

\section{Discussion}

According to previous studies, the trend of cervical cancer incidence remained consistent from 2003 to 2007. When compared with the year 2003, the crude incidences of cervical cancer in urban and rural areas increased by $70.03 \%$ and $51.00 \%$, respectively (13). In the present study, the crude incidence of cervical cancer in China in 2013 was $15.17 / 100,000$ and the ASIRC was 10.30/100,000, which was higher than that reported during 2003 to 2007. Moreover, the incidence rate increased by $52.29 \%$ when compared with 2009 (ASIRC: 7.42/100,000) (14). This could be explained by several reasons including aging of the national population, unhealthy lifestyles, and public health

Table 2 Incidence of cervical cancer in China, 2013

\begin{tabular}{lcccccc}
\hline Area & New cases $\left(\times 10^{4}\right)$ & Crude rate $\left(1 / 10^{5}\right)$ & Ratio $(\%)$ & ASIRC (1/105) & $\begin{array}{c}\text { ASIRW (1/105) } \\
\text { Cumulative rate } \\
0-74(\%)\end{array}$ \\
\hline All areas & 10.07 & 15.17 & 6.16 & 11.30 & 10.30 & 1.06 \\
Urban areas & 5.59 & 15.62 & 5.84 & 11.12 & 10.08 & 1.02 \\
Rural areas & 4.48 & 14.65 & 6.62 & 11.47 & 10.54 & 1.11 \\
\hline
\end{tabular}

ASIRC, age-standardized incidence based on the Chinese standard population; ASIRW, age-standardized incidence based on world standard population.

Table 3 Mortality rate of cervical cancer in China, 2013

\begin{tabular}{lcccccc}
\hline Area & Death $\left(\times 10^{4}\right)$ & Crude rate (1/105) & Ratio (\%) & ASMRC (1/105) & $\begin{array}{c}\text { ASMRW (1/105) } \\
\text { Cumulative rate } \\
0-74(\%)\end{array}$ \\
\hline All areas & 2.64 & 3.98 & 3.21 & 2.76 & 2.62 & 0.29 \\
Urban areas & 1.38 & 3.85 & 3.10 & 2.56 & 2.41 & 0.26 \\
Rural areas & 1.26 & 4.14 & 3.34 & 2.99 & 2.87 & 0.33 \\
\hline
\end{tabular}

ASMRC, age-standardized mortality rate based on Chinese standard population; ASMRW, age-standardized mortality rate based on world standard population. 


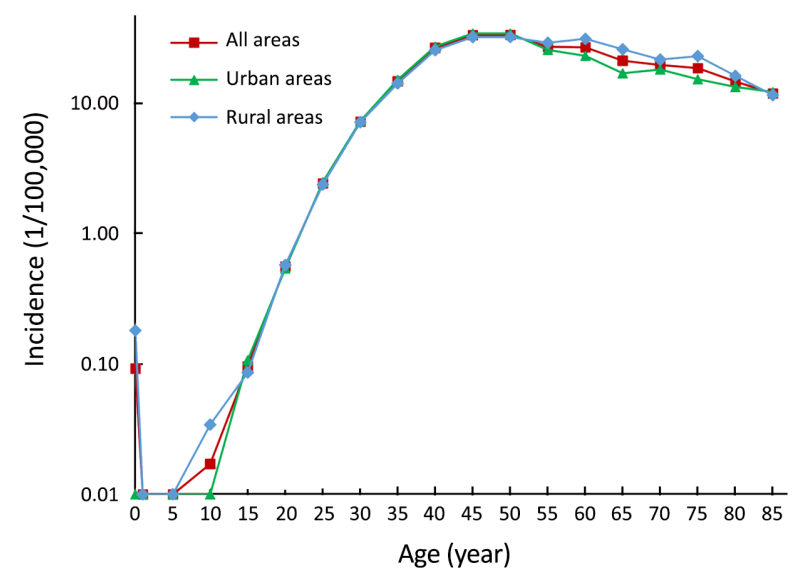

Figure 1 Age-specific incidence of cervical cancer in China, 2013.

programs (15). In addition, with the recent reform in China, national diversification of information, changes in ideas, and unsafe sexual behaviors may have led to an increase in HPV infection opportunities $(16,17)$. Thus, the incidence of cervical cancer has been increasing annually. In order to prevent cervical cancer, the National Cervical Cancer Screening Program in Rural Areas launched by the government of China has been applied to women. Unfortunately, the large population coverage required and inequities of the technology yielded unsatisfactory benefits $(18,19)$. Nonetheless, active prevention, early detection, and reasonable treatment are still important principles in cancer prevention and treatment (20).

The mortality rate of cervical cancer in China increased annually from 2003 to 2007 . The mortality rate of cervical cancer in urban and rural areas was increased by $32.43 \%$ and $3.01 \%$, respectively, in 2007 when compared to 2003 (13). The results of this study showed that the mortality rate of cervical cancer in the national cancer registration area in 2013 was 3.98/100,000, and the ASMRC was $2.76 / 100,000$. This mortality rate in 2013 was higher than that in 2009 (mortality rate: 3.28/100,000; ASMRC: $1.64 / 100,000)(14)$. Research shows that it is reasonable for a high incidence to be followed by high mortality rates: with the advances in diagnostic approaches and health awareness, new cases of cervical cancer are more frequently detected at the early stage $(21,22)$.

The incidence of cervical cancer in rural areas $(3.98 / 100,000)$ was lower than that in urban areas $(15.62 / 100,000)$; however, the mortality rate of cervical cancer in rural areas $(4.14 / 100,000)$ was higher than that in urban areas $(3.85 / 100,000)$. The high mortality rate of cervical cancer in rural areas may be related to the lack of

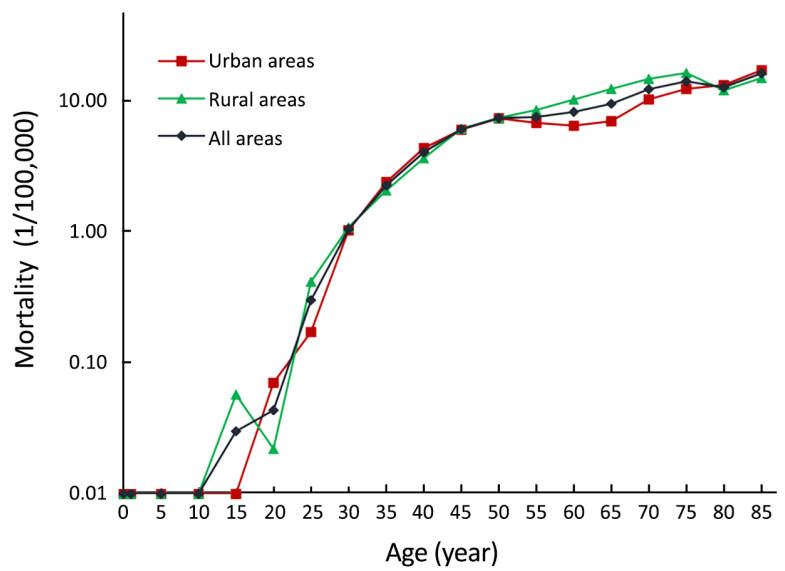

Figure 2 Age-specific mortality of cervical cancer in China, 2013.

medical resources, poor diagnosis and treatment conditions, low income, and treatment delays (22-24). In addition, the high incidence of cervical cancer in urban areas may be associated with the rapid economic development, being more receptive to new concepts, and changes in sexual behavior leading to increased opportunities for HPV infection.

In our study, the incidence of cervical cancer peaked at group of age $45-54$ years, and the mortality rate peaked at age $\geq 85$ years. The incidence of HPV-positivity has steadily increased with age in China, peaking at 31-40 years (25). The period from HPV infection to carcinogenesis could contribute to the age at which incidence peaked. The relationship between HPV infection and cervical cancer has been identified by several laboratory and epidemiological investigations $(26,27)$. HPV vaccination in high-income countries resulted in dramatic decreases in the incidence of HPV infection and associated cervical diseases (28). Our data could thus serve as guidelines to identify which patients as well as the proportion of those patients who would benefit from cervical cancer prevention and management efforts.

\section{Conclusions}

China is actively carrying out cervical cancer screening programs nationwide. However, the current incidence and mortality rates of cervical cancer continue to increase (2931). Targeting the cause of cervical cancer could help prevent new cases $(32,33)$. The cervical cancer prevention and control strategies should be tailored to the local conditions in the urban and rural areas. Efforts to promote primary prevention of cervical cancer have been carried out 
in urban areas because of the high incidence of cervical cancer. The residents of urban areas may maintain a healthy lifestyle owing to the higher average education levels, and thus avoid risk factors of cervical cancer, thereby leading to a reduced incidence of cervical cancer. The high mortality rates of cervical cancer observed in rural areas require secondary and tertiary prevention programs, which involve early detection, early diagnosis, and early treatment, such as the National Cervical Cancer Screening Program in Rural Areas. Another approach is to effectively integrate urban and rural medical resources (34). These methods could improve the level of public health and enhance the diagnosis and treatment of cervical cancer in rural areas. Moreover, these efforts count as tertiary prevention, which can extend the life of patients with cervical cancer and reduce cervical cancer mortality.

\section{Acknowledgements}

This work was supported by CAMS Innovation Fund for Medical Sciences (CIFMS) (Grant No. 2016-12M-2-004) and Ministry of Science and Technology of China (Grant No. 2014FY121100).

\section{Footnote}

Conflicts of Interest: The authors have no conflicts of interest to declare.

\section{References}

1. Ferlay J, Shin HR, Bray F, et al. Estimates of worldwide burden of cancer in 2008: GLOBOCAN 2008. Int J Cancer 2010;127:2893-917.

2. Lewis DR, Chen HS, Cockburn MG, et al. Early estimates of SEER cancer incidence, 2014. Cancer 2017;123:2524-34.

3. Wang J, Bai Z, Wang Z, et al. Comparison of secular trends in cervical cancer mortality in China and the United States: an age-period-cohort analysis. Int J Environ Res Public Health 2016;13. pii: E1148.

4. Ginsburg O, Bray F, Coleman MP, et al. The global burden of women's cancers: a grand challenge in global health. Lancet 2017;389:847-60.

5. Melan K, Janky E, Macni J, et al. Epidemiology and survival of cervical cancer in the French West-Indies: data from the Martinique Cancer Registry (20022011). Glob Health Action 2017;10:1337341.
6. Vincerzevskiene I, Jasilionis D, Austys D, et al. Education predicts cervical cancer survival: a Lithuanian cohort study. Eur J Public Health 2017;27:421-4.

7. Niccolai LM, Meek JI, Brackney M, et al. Declines in $\mathrm{HPV}$-associated high-grade cervical lesions after introduction of HPV vaccines in Connecticut, US, 2008-2015. Clin Infect Dis 2017.

8. Chen W. Cancer statistics: updated cancer burden in China. Chin J Cancer Res 2015;27:1.

9. Registry TNCC. Guideline for Chinese Cancer Registration. Beijing: Peking Union Medical College Press, 2004:48-50.

10. Curado MP, Edwards B, Shin HR, et al. Cancer incidence in five continents, Volume IX. Lyon: IARC Scientific Publications, 2008.

11. Bray F, Parkin DM. Evaluation of data quality in the cancer registry: principles and methods. Part I: comparability, validity and timeliness. Eur J Cancer 2009;45:747-55.

12. Ferlay J, Burkhard C, Whelan S, et al. Check and conversion programs for cancer registries (IARC/ IACR Tools for cancer Registries). IARC technical Report. Lyon: IARC, 2005.

13. Li N, Zheng RS, Zhang SW, et al. An analysis of incidence and mortality of cervical cancer in China, 2003-2007. Zhongguo Zhong Liu (in Chinese) 2012;21:801-4.

14. Ying Q, Xia QM, Zheng RS, et al. An analysis of incidence and mortality of cervical cancer in China, 2009. Zhongguo Zhong Liu (in Chinese) 2013;22:612-6.

15. Bode AM, Dong Z, Wang H. Cancer prevention and control: alarming challenges in China. Natl Sci Rev 2016;3:117-27.

16. Wu RF, Dai M, Qiao YL, et al. Human papillomavirus infection in women in Shenzhen City, People's Republic of China, a population typical of recent Chinese urbanisation. Int J Cancer 2007;121:1306-11.

17. Wagner M, Bennetts L, Patel H, et al. Global availability of data on HPV genotype-distribution in cervical, vulvar and vaginal disease and genotypespecific prevalence and incidence of HPV infection in females. Infect Agent Cancer 2015;10:13.

18. Di JL, Rutherford S, Wu JL, et al. Knowledge of 
cervical cancer screening among health care workers providing services across different socio-economic regions of China. Asian Pac J Cancer Prev 2016;17:2965-72.

19. Luo XM, Song L, Wu JL, et al. Analysis of the reported data of national rural cervical cancer screening project from 2012 to 2013, China. Zhonghua Yu Fang Yi Xue Za Zhi (in Chinese) 2016;50:346-50.

20. Practice Bulletin No. 168 Summary: Cervical Cancer Screening and Prevention. Obstet Gynecol 2016;128:923-5.

21. Patra S, Upadhyay M, Chhabra P. Awareness of cervical cancer and willingness to participate in screening program: Public health policy implications. J Cancer Res Ther 2017;13:318-23.

22. Koroukian SM, Bakaki PM, Htoo PT, et al. The Breast and Cervical Cancer Early Detection Program, Medicaid, and breast cancer outcomes among Ohio's underserved women. Cancer 2017;123:3097-106.

23. Kweon SS, Kim MG, Kang MR, et al. Difference of stage at cancer diagnosis by socioeconomic status for four target cancers of the National Cancer Screening Program in Korea: Results from the Gwangju and Jeonnam cancer registries. J Epidemiol 2017;27: 299-304.

24. Caldwell JT, Ford CL, Wallace SP, et al. Intersection of living in a rural versus urban area and race/ ethnicity in explaining access to health care in the United States. Am J Public Health 2016;106:1463-9.

25. Tang Y, Zheng L, Yang S, et al. Epidemiology and genotype distribution of human papillomavirus (HPV)

Cite this article as: Song B, Ding C, Chen W, Sun H, Zhang $M$, Chen $W$. Incidence and mortality of cervical cancer in China, 2013. Chin J Cancer Res 2017;29(6):471476. doi: 10.21147/j.issn.1000-9604.2017.06.01 in Southwest China: a cross-sectional five years study in non-vaccinated women. Virol J 2017;14:84.

26. Park JY, Ngan HY, Park W, et al. Asian Society of Gynecologic Oncology International Workshop 2014. J Gynecol Oncol 2015;26:68-74.

27. Walboomers JM, Jacobs MV, Manos MM, et al. Human papillomavirus is a necessary cause of invasive cervical cancer worldwide. J Pathol 1999;189:12-9.

28. Aranda S, Berkley S, Cowal S, et al. Ending cervical cancer: A call to action. Int J Gynaecol Obstet 2017;138 Suppl 1:4-6.

29. Chen $W$, Zheng R, Zeng H, et al. Annual report on status of cancer in China, 2011. Chin J Cancer Res 2015;27:2-12.

30. Chen $W$, Zheng R, Zuo T, et al. National cancer incidence and mortality in China, 2012. Chin J Cancer Res 2016;28:1-11.

31. Chen $\mathrm{W}$, Zheng R, Zhang S, et al. Cancer incidence and mortality in China in 2013: an analysis based on urbanization level. Chin J Cancer Res 2017;29:1-10.

32. Lea JS, Lin KY. Cervical cancer. Obstet Gynecol Clin North Am 2012;39:233-53.

33. Haggar FA, Preen DB, Pereira G, et al. Cancer incidence and mortality trends in Australian adolescents and young adults, 1982-2007. BMC Cancer 2012;12:151.

34. Woo RK, Skarsgard ED. Innovating for quality and value: Utilizing national quality improvement programs to identify opportunities for responsible surgical innovation. Semin Pediatr Surg 2015;24: $138-40$. 